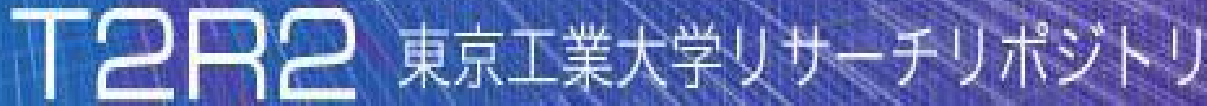

\section{Tokyo Tech Research Repository}

\section{論文 /著書情報 \\ Article /Book Information}

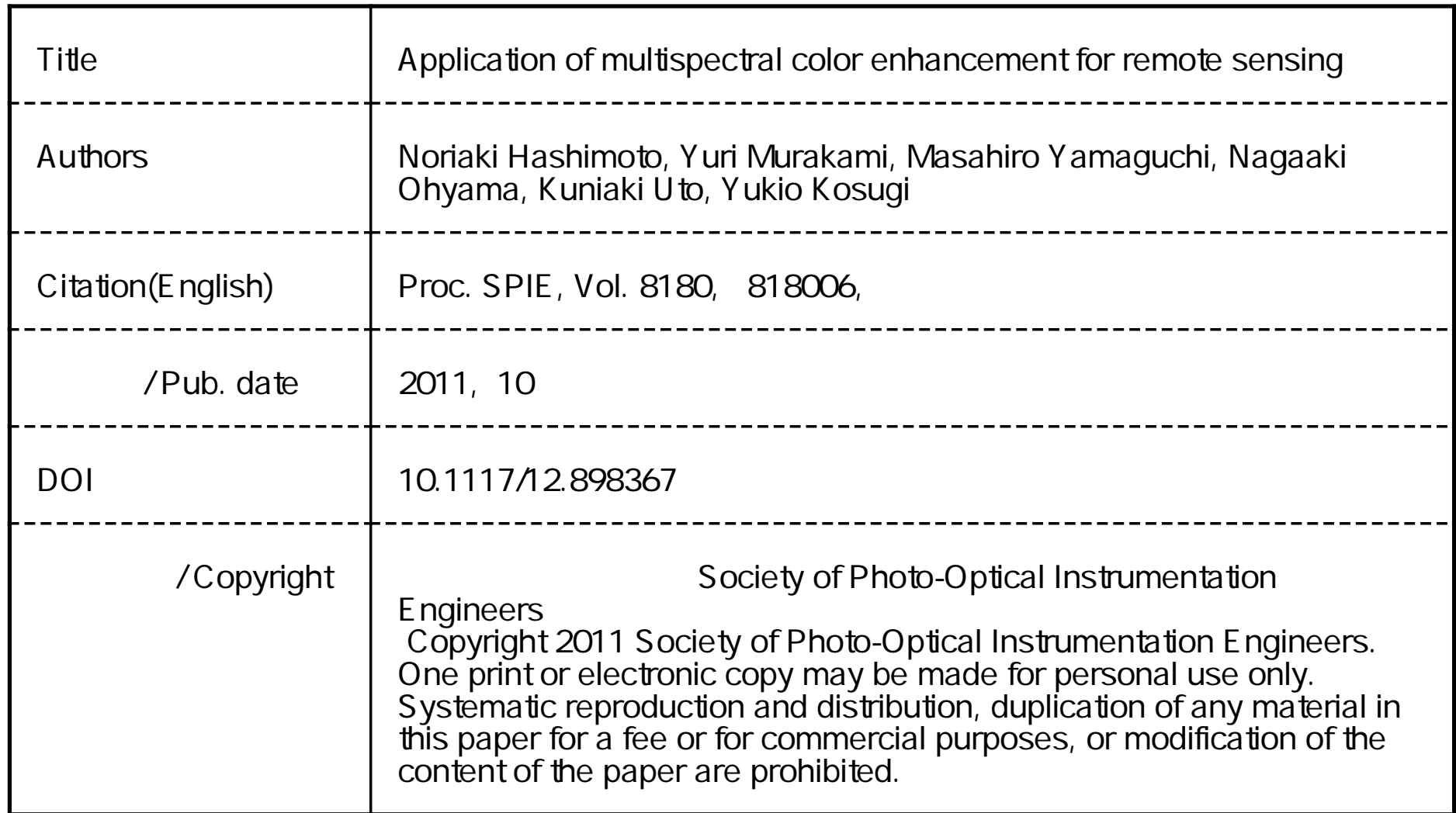




\title{
Application of multispectral color enhancement for remote sensing
}

\author{
Noriaki Hashimoto*a, Yuri Murakami ${ }^{\mathrm{b}}$, Masahiro Yamaguchic, Nagaaki Ohyama ${ }^{\mathrm{b}}$, Kuniaki Uto ${ }^{\mathrm{a}}$, \\ Yukio Kosugi ${ }^{\mathrm{a}}$ \\ ${ }^{a}$ Interdisciplinary Graduate School of Science and Engineering, Tokyo Institute of Technology, \\ 4259 Nagatsuta-cho, Midori-ku, Yokohama 226-8502, Japan; \\ ${ }^{\mathrm{b}}$ Imaging Science and Engineering Laboratory, Tokyo Institute of Technology, \\ 4259 Nagatsuta-cho, Midori-ku, Yokohama 226-8503, Japan; \\ ${ }^{c}$ Global Scientific Information and Computing Center, Tokyo Institute of Technology, \\ 2-12-1 O-okayama, Meguro-ku, Tokyo 152-8550, Japan
}

\begin{abstract}
Previously, we proposed a color enhancement method, which enhances the spectral features in multispectral images without changing the average color distribution, since the natural color of the object is sometimes important. In the proposed method, a user can specify both the spectral band to extract the spectral feature and the color to be visualized, respectively. This method can visualize spectral features even if the wavelength of the specified spectral band is in nonvisible range or the image has a large number of spectral bands. That is, the enhancement method is also effective for hyperspectral images which are often used in remote sensing applications. If we can find the meaningful spectral features in them, those features might be employed as novel indices. In this paper, we apply the enhancement method to hyperspectral images of rice paddy and trees. We observed the enhanced results of these images, and could find the spectral features which might be useful to discriminate different species and even the different conditions in the same species. The results showed that these features might be utilized for novel indices or other applications in remote sensing.
\end{abstract}

Keywords: Image processing, color enhancement, multispectral and hyperspectral imaging, remote sensing, principal component analysis

\section{INTRODUCTION}

Spectral imaging, which is an imaging technique with more than 3 color channels, is useful for color reproduction, image analysis and so on [1-4]. Especially, many studies in remote sensing have employed multispectral or hyperspectral images for image analysis such as classification, pattern recognition, spectral unmixing and etc. for a long time [5-8]. Since spectral features, which cannot be seen in RGB images, are included in them, many methods to visualize their spectral features have been developed. Previously, a multispectral color enhancement method, which can enhance the spectral features in multispectral images without changing the average color distribution, as the natural color of the object is sometimes important [9]. In this method, the differences between the original multispectral image and its approximation by a few principal components at specified spectral bands are amplified. Then, the indiscernible spectral feature in the multispectral image is visualized without changing the average color distribution. However, this enhancement method could not visualize the spectral features in non-visible range or in the case the image has a large number of spectral bands such like hyperspectral images. Therefore, authors extended the enhancement method and our enhancement method can visualize spectral features in the image effectively in such cases [10].

On the other hand, hyperspectral images, which are often used in remote sensing for image analysis, generally include non-visible range such as infrared and have more than 100 color channels. The proposed enhancement method is effective even for hyperspectral images and we also confirmed the validity of the proposed method for hyperspectral images by using a rice paddy image in Ref. 10. It is expected to apply the proposed method to explore meaningful spectral features in hyperspectral images for remote sensing, for the development of novel indices.

In this paper, we applied the proposed enhancement method to 101-band hyperspectral images of a rice paddy and trees, and investigated its effectiveness in remote sensing application. The enhancement method was applied to each

*hashimoto.n.ac@m.titech.ac.jp; phone 8145 924-5183; fax 8145 924-5175;

Image and Signal Processing for Remote Sensing XVII, edited by Lorenzo Bruzzone,

Proc. of SPIE Vol. 8180, 818006 - @ 2011 SPIE · CCC code: 0277-786X/11/\$18 · doi: 10.1117/12.898367

Proc. of SPIE Vol. $8180818006-1$ 
wavelength and the enhanced images were observed. When we found the interested region in the enhanced image, we extracted the spectral reflectance in the image and analyzed their spectra and spectral features. In the results, we showed the capability that the spectral features found by the enhancement method could be helpful for the development of novel indices.

This paper is organized as follows: In Section 2, our enhancement algorithm is reviewed. In Section 3, we show the experimental results in the application of the proposed method to hyperspectral images of rice paddy and trees, and finally close with conclusions in Section 4.

\section{MULTISPECTRAL COLOR ENHANCEMENT}

\subsection{Multispectral color enhancement algorithm}

The color enhancement used in this paper is proposed by the authors [10]. This method enhances the color difference from dominant Karhunen-Loeve (KL) components without changing the color determined by the dominant components. First, a set of spectral data is extracted from the image in order to derive the dominant component. The data can be extracted from the entire image, or from part of the image (e.g. background region), depending on the requirement of the application. Then, a covariance matrix is derived from the extracted spectral samples to make the KL basis vectors. The first few KL vectors are used to estimate the dominant component of the image. In the $N$-band multispectral image, the enhanced signal value vector for $j$-th pixel $\mathbf{g}_{\mathrm{e} j}(N$-dimensional vector) is represented as,

$$
\mathbf{g}_{\mathrm{e} j}=\mathbf{W}\left(\mathbf{g}_{j}-\mathbf{s}_{j}\right)+\mathbf{g}_{j},
$$

where $\mathbf{W}$ is an $N \times N$ matrix for the enhancement, $\mathbf{g}_{j}$ is the original multispectral signal value of $j$-th pixel and $\mathbf{s}_{j}$ is the signal value estimated with $m-\mathrm{KL}$ vectors $(m<N)$ written as,

$$
\mathbf{s}_{j}=\sum_{i=1}^{m} \alpha_{i j} \mathbf{u}_{i}+\overline{\mathbf{g}},
$$

where $\mathbf{u}_{i}$ is $i$-th KL basis vector ( $N$-dimensional vector) and $\overline{\mathbf{g}}$ is the average vector of the set of pixel data for $\mathrm{KL}$ basis vectors; $\mathbf{g}_{j}-\mathbf{s}_{j}$ is considered to be a residual component. Furthermore, $\alpha_{i j}$ is $i$-th KL coefficient for $j$-th pixel expressed as,

$$
\alpha_{i j}=\mathbf{u}_{i}^{T}\left(\mathbf{g}_{j}-\overline{\mathbf{g}}\right)
$$

In Ref. 10, $q$-th column vector of the matrix $\mathbf{W}$ is defined as follows,

$$
[\mathbf{W}]_{q}=\left\{\begin{array}{cc}
k\left(\mathbf{g}_{\mathrm{d}}-\mathbf{g}_{\mathrm{a}}\right) & q=n \\
\mathbf{0} & \text { otherwise }
\end{array},\right.
$$

where $\mathbf{g}_{\mathrm{d}}$ is the spectral data of the target color for visualization and $\mathbf{g}_{\mathrm{a}}$ is the spectral data of the background in the image. We can use the average spectral data of the entire image as the background $\mathbf{g}_{\mathrm{a}}$ based on the assumption the color distribution in the image is similar. The enhanced image $\mathbf{g}_{\mathrm{e} j}$ is converted into the RGB color image by using a colormatching function (CMF) such as CIE $1931 \mathrm{XYZ} \mathrm{CMF}$, an illumination spectrum and a matrix for XYZ to RGB transform.

\section{EXPERIMENTS}

\subsection{Imaging system and determination of target color}

The images used in following experiments were captured by using a cargo crane with the hyperspectral sensor, 
ImSpector V10 made by Specim Co., which has 121 bands from 400-1000 nm, $3 \mathrm{~nm}$ of spectral resolution, and $5 \mathrm{~nm}$ of sampling interval [11]. However, the components of the longer wavelengths than $900 \mathrm{~nm}$ were not used since they included much noise. Each pixel value in the image was transformed into spectral reflectance with reference to the pixel value of the standard white board placed in the same image.

And we determined the target spectrum $\mathbf{g}_{\mathrm{d}}$ in Eq. (4) by selecting from Macbeth Color Checker image shown in Fig. 1. This is a physical object which was obtained with the same hyperspectral sensor. Because most of the images used in these experiments consist of green, we utilized the spectrum of "magenta" arrowed in the figure as the spectrum $\mathbf{g}_{\mathrm{d}}$.

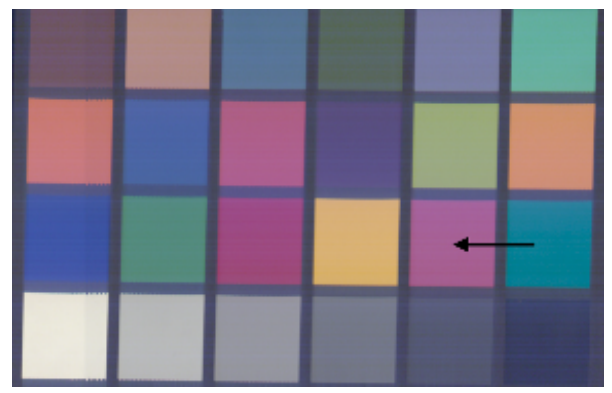

Figure 1. The natural color representation of macbeth color checker image with D65 illumination. The arrow in the image indicates the color "magenta" used in experiments.

\subsection{Application to rice paddy images}

First, the enhancement method was applied to rice paddy images. Figure 2 is the rice paddy images captured on (a) Aug. and (b) Sept. (after heading), 2007, respectively.

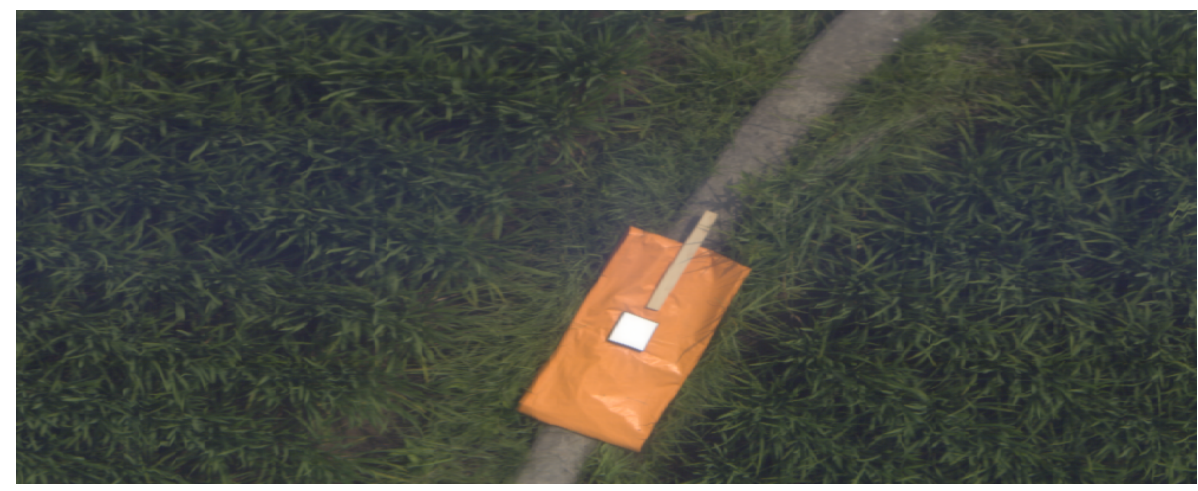

(a)

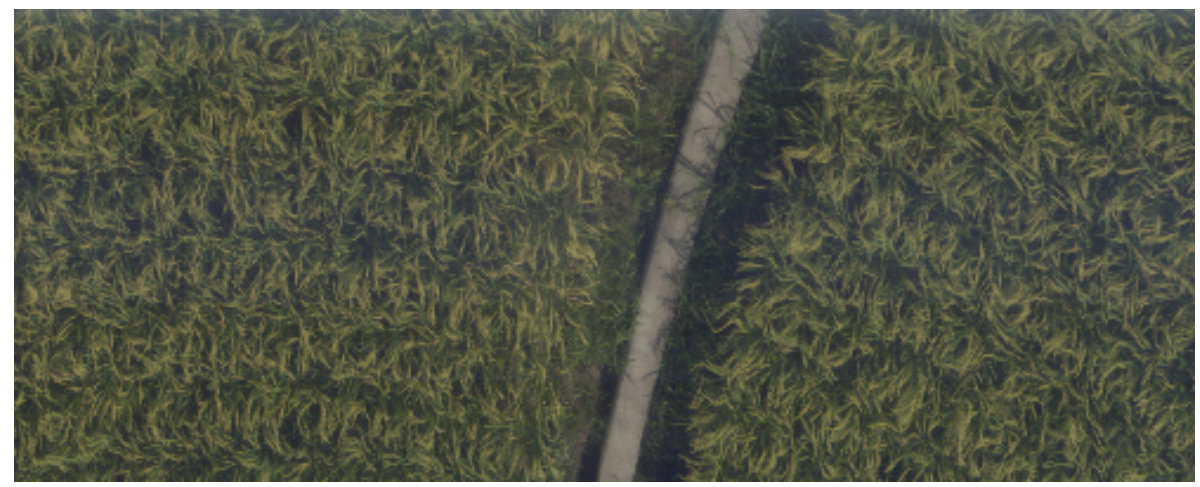

(b)

Figure 2. The natural color representation of rice paddy images. (a) was captured on Aug. and (b) was on Sept., 2007. 
The image sizes are $1,000 \times 400$ pixels and these images have 121 spectral bands (but the longer 20 bands were not used as mentioned above). In Ref. 10, we could find the spectral feature useful for discrimination between the crop region and the weed region, that is, the species of plants. Here, we applied the proposed method to these images and explored the spectral features caused by the difference of growing level in a single species. A part of the image captured on Aug. $(200 \times 400$ pixels in the left side) was used to generate the KL basis vectors. If the average reflectance between $750-900$ $\mathrm{nm}$ was less than 0.3 in each pixel, that pixel was regarded as the background and not used for principal component. In addition, those pixels were also ignored at the enhancement process. In this experiment, we used three KL basis vectors, because we knew that only the first KL basis vector cannot represent the spectra used for generating KL basis vectors well from the previous results [10].

Under these conditions, we enhanced the both rice paddy images in the bands from 400 to $900 \mathrm{~nm}$ at $10 \mathrm{~nm}$ sampling interval and investigated their results. In the enhanced results at $650 \mathrm{~nm}$, as you can see in Fig. 3, only the image captured on Sept. was enhanced in the crop region with magenta color. Furthermore, some regions were remaining with green color as shown in Fig. 4. Then, we selected the region by manual and extracted the spectra of the crop on Aug. "CropAug", the enhanced crop "CropSept_en" (with magenta in Fig. 4) and not-enhanced crop "CropSept" (with green in Fig. 4) on Sept, respectively. The original spectra of selected regions and the residual components of them with three KL basis are shown in Fig. 5. In this graph, "CropSept" has the similar shape of spectrum to "CropAug". It can be thought that "CropAug" indicates leaves because this image was captured before heading. Therefore, "CropSept_en" is considered to be the ear region, as the image captured on Sept. must have two classes because of heading. The ear region is not differentiated in the original image, but we can see them easily by the color enhancement method. This result showed the capability that the enhancement results change depending on the growing levels. As shown in Fig. 5(b), the ear region has large residual components between 600-700 nm compared with leaves. Using their spectral feature, we could see the difference of growing levels visually and it was useful for novel vegetation indices by selecting the appropriate wavelength for the color enhancement.

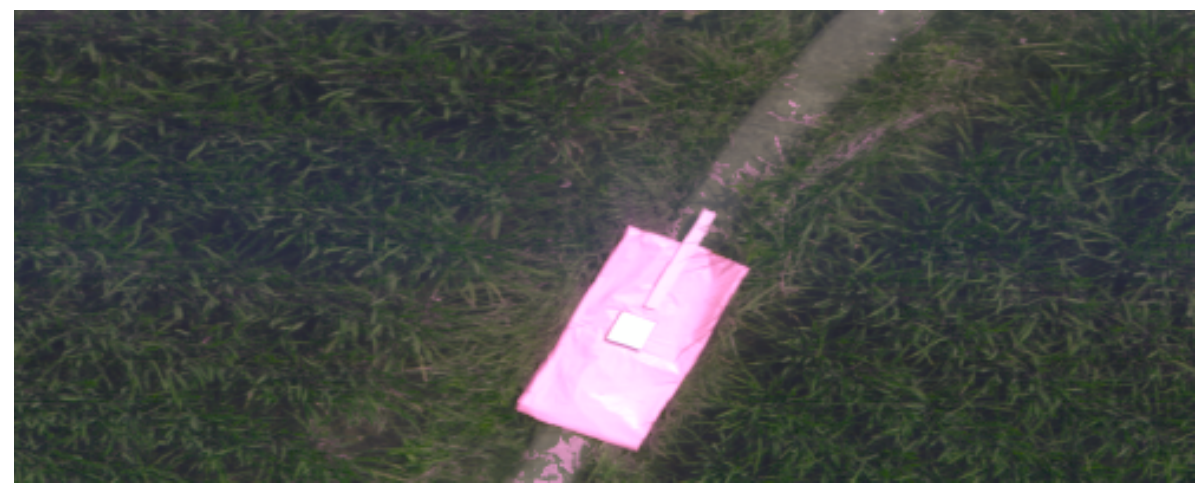

(a)

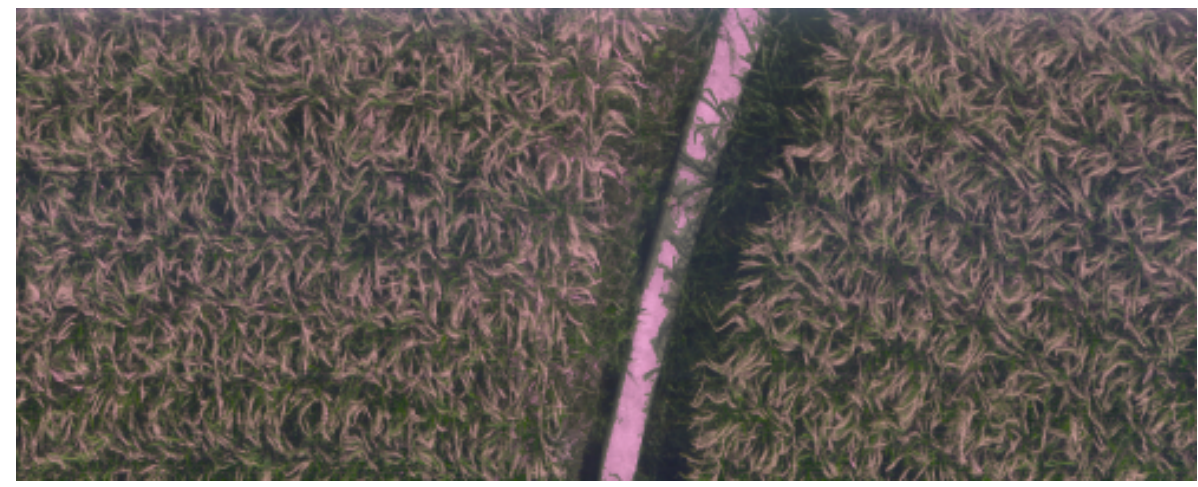

(b)

Figure 3. The enhanced results of rice paddy images at $650 \mathrm{~nm}(k=10)$. (a) is the result of the image captured on Aug. and (b) is the result of the image captured on Sept. 


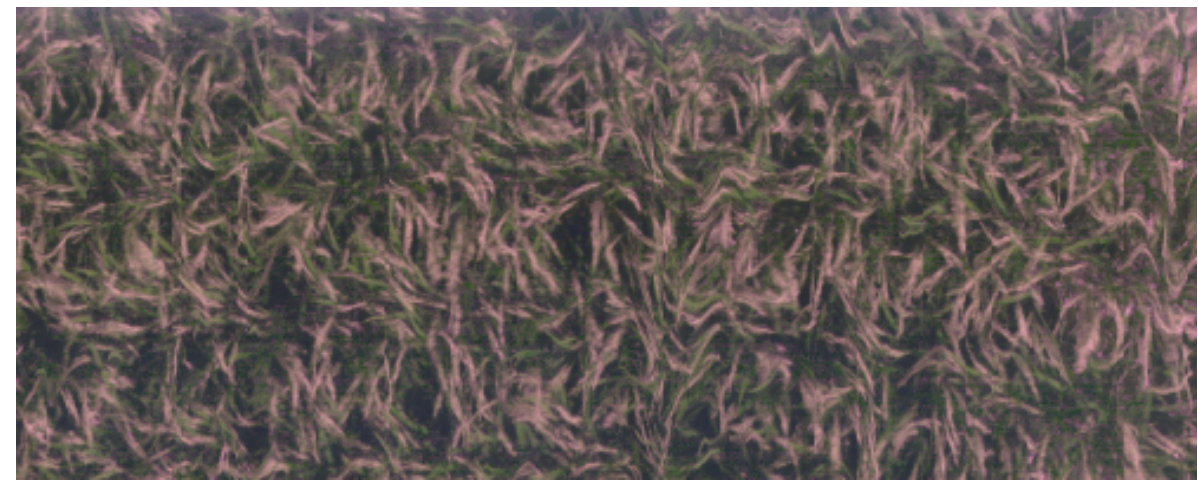

Figure 4. $500 \times 200$ pixels cropped from the magnified version of the enhanced image [Fig. 3(b)].

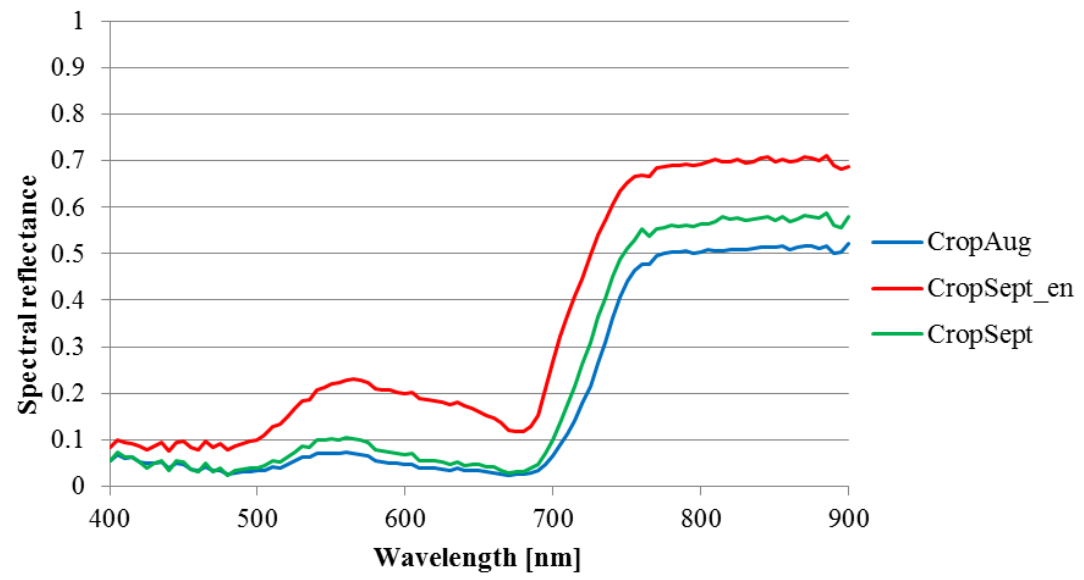

(a)

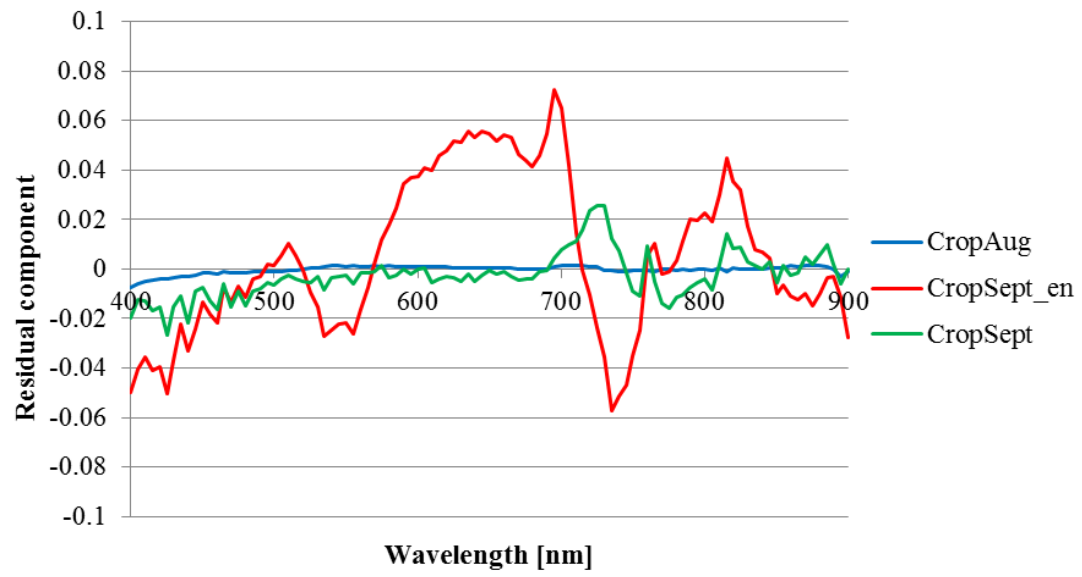

(b)

Figure 5. The spectral data of selected regions in the rice paddy images. (a) is the original spectra and (b) is the residual components with three KL basis vectors. Each plot represents the average of 50 samples.

\subsection{Application to tree images}

Next, we applied the proposed enhancement method to tree image. Figure 6 shows the images used in this experiment; cypress (Chamaecyparis obtusa) and elm (Ulmus davidiana var. japonica) are included in Fig. 6(a), oak (Quercus 
crispula) and beech (Fagus crenata) are in Fig. 6(b), respectively. Their image sizes are $1000 \times 350$ pixels [Fig. 6(a)] and $900 \times 300$ pixels [Fig. 6(b)]. We employed the image shown in Fig. 6(a) for generating KL basis vectors and used the other image as the test image for the enhancement. Similarly to the last experiment, the pixels with low spectral reflectance in the longer wavelengths were ignored for each process. The image, which includes oak and beech, was enhanced in all wavelengths at $5 \mathrm{~nm}$ interval with three KL basis vectors. The enhanced result at $695 \mathrm{~nm}$ is shown in Fig. 7.

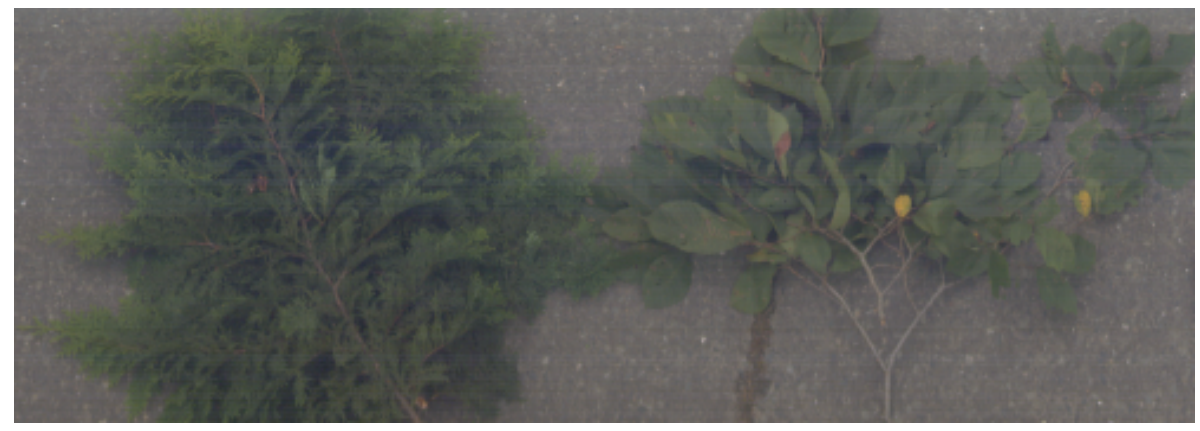

(a)

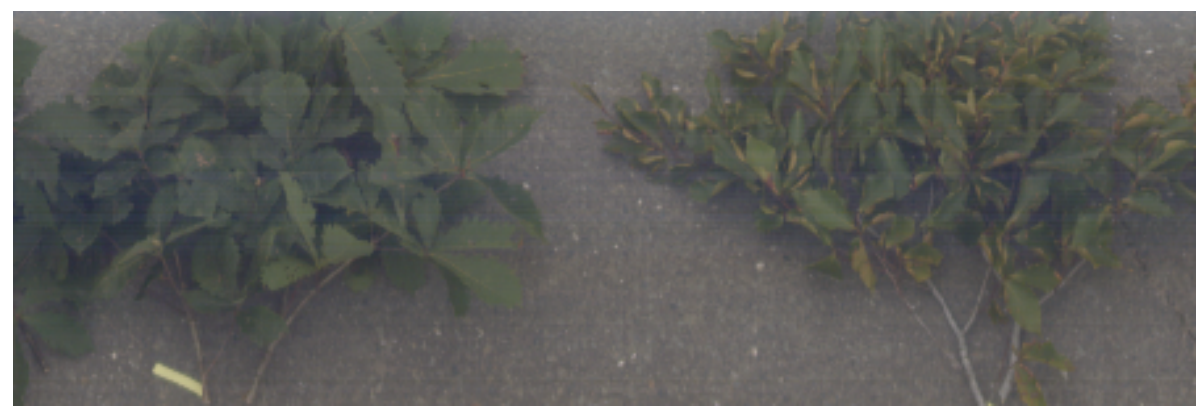

(b)

Figure 6. The natural color representation of tree images. (a) is the reference image including cypress (left) and elm (right), and (b) is the test image with oak (left) and beech (right).

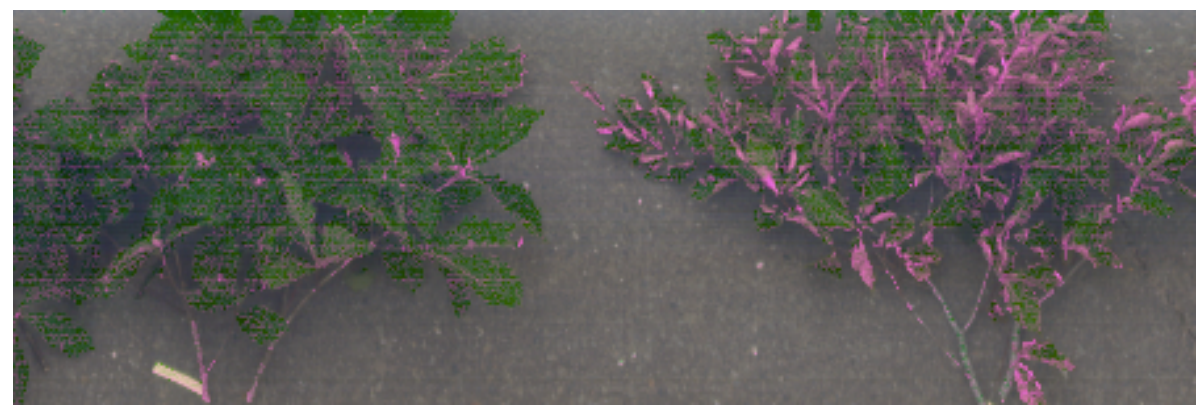

Figure 7. The enhanced result of tree image at $695 \mathrm{~nm}(k=30)$.

In this result, a part of beech leaves was enhanced with magenta color. Then, we selected the pixels by manual and picked the spectra of the oak "Oak", the enhanced beech "Beech_en" (with magenta in Fig. 7) and not-enhanced beech "Beech" (with green in Fig. 7), respectively. The original spectra of selected regions and the residual components of them with three KL basis are shown in Fig. 8. In Fig. 8(a), we can observe the blue-shift of the red-edge in "Beech_en", and this region is considered to be the back of leaves or water stressed. From this result, the enhancement method could visualize the condition of leaves and was useful for the early detection of tree diseases. In addition, beech regions have large residual errors in $705 \mathrm{~nm}$ with regardless of their leaves' condition in Fig. 8(b). We can confirm those features as the enhanced image in Fig. 9. This result showed that the spectral features found by the enhancement method might be 
applied for tree discrimination. The capability of discrimination between oak and beech is significantly valuable from the aspect of oak wilt. As shown above, the visualization of spectral features make it easier to explore spectral features in the image and the spectral feature found in the experiments could be useful for other image analysis.

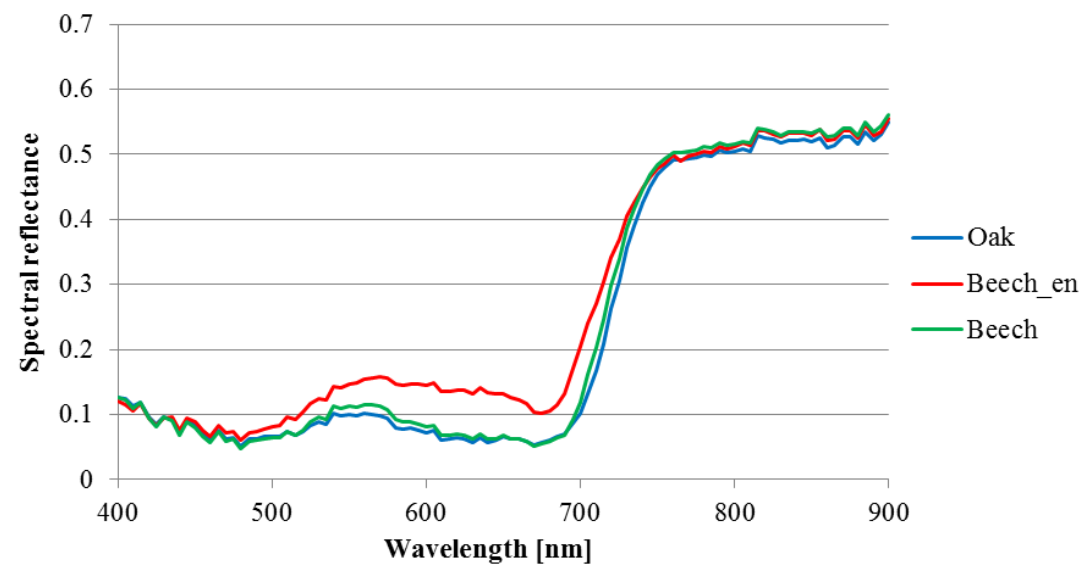

(a)

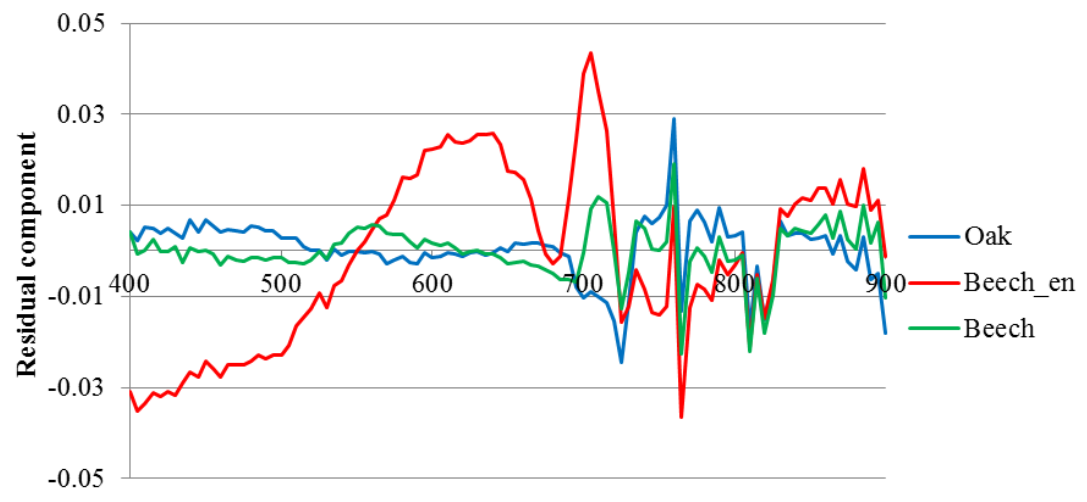

Wavelength $[\mathrm{nm}]$

(b)

Figure 8. The spectral data of selected regions in the tree image. (a) is the original spectra and (b) is the residual components with three KL basis vectors. Each plot represents the average of 50 samples.

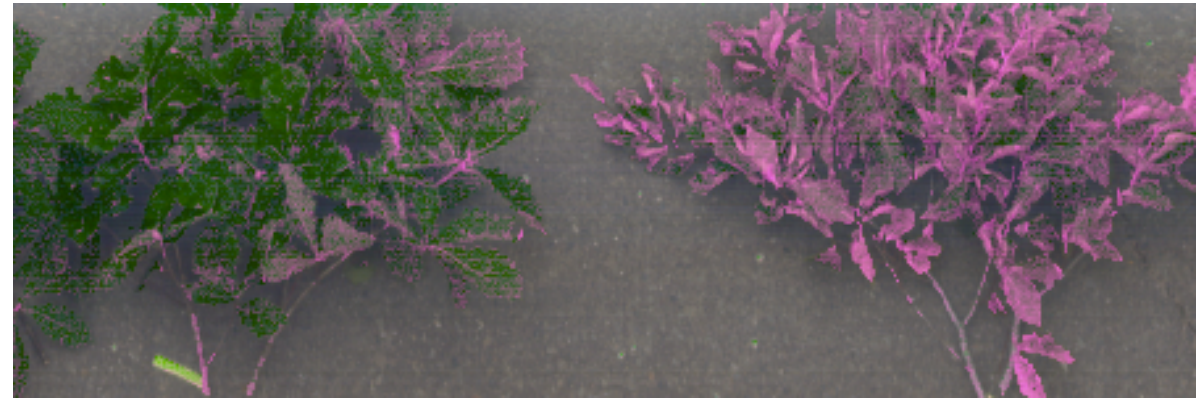

Figure 9. The enhanced result of tree image at $705 \mathrm{~nm}(k=30)$. 


\section{CONCLUSIONS}

In this paper, we applied the multispectral color enhancement to hyperspectral images for remote sensing. The enhancement method can visualize the spectral features in the image without changing the average color distribution and this makes it easier to explore the spectral features. We used the hyperspectral images of rice paddy and trees, and could find the spectral features which might be useful to discriminate different species or regions with different conditions in the same species. It is considered that these spectral features found in these experiments might be utilized for novel indices or other application in remote sensing.

As a future work, we investigate the relationship between these results and physical meanings. And we'd like to apply the enhancement method to other images which are not limited to remote sensing region.

\section{REFERENCES}

[1] Yamaguchi, M., Haneishi, H. and Ohyama, N., "Beyond red-green-blue (RGB): Spectrum-based color imaging technology," J. Imaging Sci. Technol. 52(1), 010201-1-15 (2008).

[2] Hardeberg, J. Y., Schmitt, F. and Brettel, H., "Multispectral color image capture using a liquid crystal tunable filter," Opt. Eng. 41(10), 2532-2548 (2002).

[3] Landgrebe, D. A., "Hyperspectral image data analysis," IEEE Signal Process. Mag. 19(1), 17-28 (2002).

[4] Levenson, R. M. and Mansfield, J. R., "Multispectral imaging in biology and medicine: Slices of life," Cytometry, 69A, 748-758 (2006).

[5] Melgani, F. and Bruzzone, L., "Classification of hyperspectral remote sensing images with support vector machines," IEEE Trans. Geosci. Remote Sens. 42(8), 1778-1790 (2004).

[6] Zhong, Y., Zhang, L., Huang, B. and Li, P., "An unsupervised artificial immune classifier for multi/hyperspectral remote sensing imagery," IEEE Trans. Geosci. Remote Sens. 44(2), 420-431 (2006).

[7] Chen, C. H. and Ho, P.-G. P., "Statistical pattern recognition in remote sensing," Pattern Recognition 41(9), 2731-2741 (2008).

[8] Nascimento, J. M. P. and Dias, J. M. B., "Vertex component analysis: A fast algorithm to unmix hyperspectral data," IEEE Trans. Geosci. Remote Sens. 43(4), 898-910 (2005).

[9] Mitsui, M., Murakami, Y., Obi. T., Yamaguchi, M. and Ohyama, N., "Color enhancement in multispectral image using the Karhunen-Loeve transform," Opt. Rev. 12(2), 69-75 (2005).

[10] Hashimoto, N., Murakami, Y., Bautista, P. A., Yamaguchi, M., Obi, T., Ohyama, N., Uto, K. and Kosugi, Y., "Multispectral image enhancement for effective visualization," Opt. Express 19(10), 9315-9329 (2011).

[11] Kosaka, N., Uto, K. and Kosugi, Y., "ICA-aided mixed-pixel analysis of hyperspectral data in agricultural land," IEEE Trans. Geosci. Remote Sens. 2(2), 220-224 (2005). 